\title{
\begin{tabular}{l|l} 
Mibraries & DSpace@MIT
\end{tabular}
}

\author{
MIT Open Access Articles
}

\section{Nonlinear Feedback Control of a Gravity-Assisted Underactuated Manipulator With Application to Aircraft Assembly}

The MIT Faculty has made this article openly available. Please share how this access benefits you. Your story matters.

Citation: Roy, B., and H.H. Asada. “Nonlinear Feedback Control of a Gravity-Assisted Underactuated Manipulator With Application to Aircraft Assembly." Robotics, IEEE Transactions on 25.5 (2009): 1125-1133. (C) Copyright 2009 IEEE

As Published: http://dx.doi.org/10.1109/tro.2009.2025067

Publisher: Institute of Electrical and Electronics Engineers

Persistent URL: http://hdl.handle.net/1721.1/60048

Version: Final published version: final published article, as it appeared in a journal, conference proceedings, or other formally published context

Terms of Use: Article is made available in accordance with the publisher's policy and may be subject to US copyright law. Please refer to the publisher's site for terms of use. 


\title{
Nonlinear Feedback Control of a Gravity-Assisted Underactuated Manipulator With Application to Aircraft Assembly
}

\author{
Binayak Roy and H. Harry Asada, Member, IEEE
}

\begin{abstract}
A nonlinear feedback scheme for a gravity-assisted underactuated manipulator with second-order nonholonomic constraints is presented in this paper. The joints of the hyper articulated arm have no dedicated actuators but are activated by gravity. By tilting the base link appropriately, the gravitational torque drives the unactuated links to a desired angular position. With simple locking mechanisms, the hyperarticulated arm can change its configuration using only one actuator at the base. This underactuated arm design was motivated by the need for a compact snake-like robot that can go into aircraft wings and perform assembly operations using heavy end-effectors. The dynamics of the unactuated links are essentially second-order nonholonomic constraints for which there are no general methods to design closed-loop control. We propose a nonlinear closed-loop control law that is guaranteed to be stable in positioning one unactuated joint at a time. We synthesize a Lyapunov function to prove the convergence of this control scheme. The Lyapunov function also generates estimates of the domain of convergence of the control law for various control gains. The control algorithm is implemented on a prototype three-link system. Finally, we provide some experimental results to demonstrate the efficacy of the control scheme.
\end{abstract}

Index Terms-Gravity assisted, nonlinear feedback control, underactuated manipulator.

\section{INTRODUCTION}

$\mathbf{R}$ OBOTS have seldom been used in aircraft manufacturing and assembly. Although aircrafts are small in lot size, numerous repetitive assembly operations have to be performed on a single aircraft. Such operations are often ergonomically challenging and result in low productivity as well as frequent injuries. Thus, there is a pressing need to shift from manual to automated robotic assembly. This is exemplified in the following wing-box assembly.

Fig. 1 shows one segment of an aircraft wing, which is called a wing box. Several assembly operations, such as burr-less drilling and fastener installations, have to be carried out inside the wing box after the upper and lower skin panels are in place. The interior of the wing box is accessible only through small portholes along its length. The portholes are roughly rectangular with

Manuscript received May 4, 2008; revised December 16, 2008 and May 30, 2009. First published July 6, 2009; current version published October 9, 2009. This paper was recommended for publication by Associate Editor S. Hirai and Editor K. Lynch upon evaluation of the reviewers' comments. This work was supported by Boeing Company.

The authors are with the d'Arbeloff Laboratory for Information Systems and Technology, Department of Mechanical Engineering, Massachusetts Institute of Technology, Cambridge, MA 02139 USA (e-mail: binayak@mit.edu; asada@mit.edu).

Color versions of one or more of the figures in this paper are available online at http://ieeexplore.ieee.org.

Digital Object Identifier 10.1109/TRO.2009.2025067

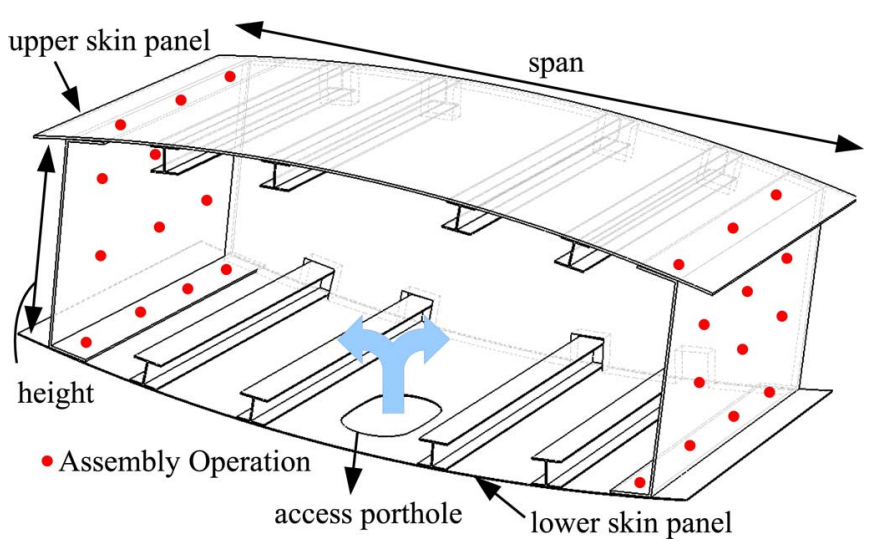

Fig. 1. Cross section of aircraft's wing box.

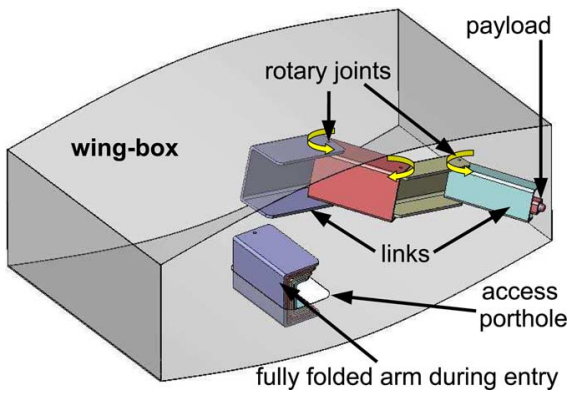

Fig. 2. Structure of robot arm.

dimensions of $45 \mathrm{~cm} \times 23 \mathrm{~cm}$. The wing box also has a substantial span, which varies from 1 to $3 \mathrm{~m}$, depending upon the size of the aircraft. The height of the wing box varies from about 20 to $90 \mathrm{~cm}$, depending on the size of the aircraft. At present, the assembly operations are carried out manually. A worker enters the wing box through the small portholes and lies flat on the base, while carrying out the assembly operations. Evidently, the working conditions are ergonomically challenging.

We have proposed a "nested-channel" serial-linkage mechanism that is capable of operating inside an aircraft's wing box [1]. The links are essentially C-channels with successively smaller base and leg lengths, as shown in Fig. 2, which are connected by 1-degree-of-freedom (DOF) rotary joints, the axes of which are parallel. The use of channel structures is advantageous for a number of reasons. The channels can fold into each other, thus resulting in an extremely compact structure during entry through the porthole, as shown in Fig. 2. Once inside the wing box, the links may be deployed to access distal points in 


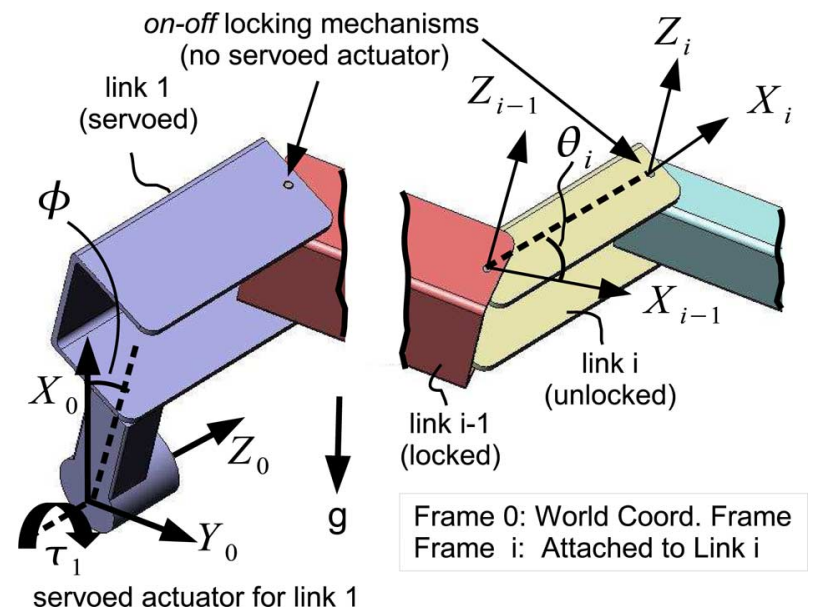

Fig. 3. Schematic of $n$-link robot arm.

the assembly space. The open-channel structure also facilitates the attachment of a payload to the last link without increasing the overall dimensions of the arm.

The lack of a compact, powerful, and high-stroke actuation mechanism is the primary bottleneck in the development of the hyperarticulated arm. In our previous work [1], we have proposed an underactuated design concept, which obviates the use of dedicated actuators for each joint. Instead, we utilize gravity to drive individual joints. This drastically reduces the size and weight of the manipulator arm. The methodology requires a single actuator to tilt the arm at the base. This single actuator can be placed outside the wing box and can be used in conjunction with simple locking mechanisms to reconfigure the serial-linkage structure.

The reconfiguration scheme is illustrated in Fig. 3, which shows a schematic of an $n$-link robot arm. The base link (link 1 ) is the only servoed link. It may be rotated about a fixed axis $Z_{0}$, which is orthogonal to the direction of gravity. All other joint axes $\left(Z_{j}, j \neq 0\right)$ are orthogonal to $Z_{0}$. They are equipped with simple binary (ON-OFF) locking mechanisms only. The goal is to rotate link $i$ about $Z_{i-1}$ by actuating link 1 appropriately. All unactuated links except link $i$ are locked. Link 1 starts in the vertical upright position. Then, it is rotated, first clockwise and then counterclockwise, before being brought back to its vertical position. This tends to accelerate, and then decelerate link $i$ due to gravity and dynamic coupling with link 1 . By controlling the tilting angle of link 1 , link $i$ can be brought to a desired position with zero velocity. Link $i$ may be locked thereafter. This procedure can be repeated sequentially for the other unactuated links. Contraction of the arm can be performed by reversing the previous deployment procedure.

Underactuated systems represent a special class of nonholonomic systems, viz., systems with second-order nonholonomic constraints. An excellent survey of general nonholonomic systems is presented in [2] and [3]. The nonholonomic nature of underactuated systems was first pointed out by Oriolo and Nakamura [4]. Since then, several researchers have considered the design and control of underactuated mechanisms for robotics applications [5]-[18]. Most of the work in this area deals with the planar (vertical or horizontal) case where the actuated and unactuated joint axes are parallel. In our approach, the actuated and unactuated joints are orthogonal, and we can modulate the effects of gravity by controlling the actuated joint. Other systems in a gravity field, like the Acrobot [19], [20], have isolated equilibrium points. Since we can modulate the effect of gravity, there exists a continuum of equilibrium points in our system. The presence of gravity also renders our system locally controllable, as can be seen from local linearization [4]. If the local linearization is controllable over the entire state space, so is the nonlinear system. This ensures that we can go from any initial point to any final point in the configuration space of the unactuated coordinate. However, it is inefficient to patch together local linear control laws to traverse the entire configuration space. Moreover, any control design must ensure that the range of motion of the actuated coordinate is small, because the arm operates inside an aircraft's wing box. Existing approaches [5], [6] to the control of underactuated systems generate constructive global control laws applied to specific systems. Such constructive control laws cannot be directly applied to our system.

In our earlier work [21], we have proposed several motionplanning algorithms suitable for the gravity-assisted underactuated robot arm. They include parameterized trajectory planning for the actuated joint and feedforward optimal control. These are open-loop techniques and work well in the absence of disturbances. Also, an exact knowledge of the system dynamics is needed. In particular, a good estimate of Coulomb friction is necessary for accurate position control. However, it is unrealistic to assume prior knowledge of such state-dependent unknown parameters. These considerations necessitate the development of a closed-loop control strategy for our system. We have presented some initial results for feedback control in [22]. The stability analysis therein was restricted to a particular choice of feedback gains and is of limited value from a theoretical as well as practical standpoint. Further, our previous work did not provide analytical estimates for the domain of convergence (DOC). Our present study addresses both issues by generalizing the stability analysis for arbitrary gains and generating analytical estimates for the DOC.

This paper is organized as follows. We first explore the system dynamics to develop an understanding of the relationship between the actuated and unactuated DOFs. We make important approximations to capture the dominant effects in the system dynamics to facilitate control design. Next, we propose a closed-loop control strategy for point-to-point control of the unactuated coordinate. We synthesize a Lyapunov function to prove the convergence of the control law. The Lyapunov function is used to generate estimates of the DOC of the control law for various control gains. Finally, we present some experimental results that demonstrate the efficacy of the control law.

\section{System Dynamics}

Fig. 3 shows a schematic of an $n$-link robot arm with one actuated (link 1) and $n-1$ unactuated links. $X_{0} Y_{0} Z_{0}$ denotes the world coordinate frame. The coordinate frames are attached according to the Denavit-Hartenberg convention with the 
$i$ th-coordinate frame fixed to the $i$ th link. We seek rotation of link $i(i \geq 2)$ about the axis $Z_{i-1}$ by rotating link 1 about the horizontal axis $Z_{0}$. The angle $\theta_{i}$ denotes the angular displacement of link $i$ relative to link $i-1$. For clarity, the displacement of the first joint (which is the only servoed joint), which is obtained by tilting link 1 relative to the fixed vertical axis $X_{0}$, is denoted by $\phi$.

The system dynamics may be written as

$$
\begin{gathered}
{\left[\begin{array}{ll}
H_{11} & H_{i 1} \\
H_{1 i} & H_{i i}
\end{array}\right]\left[\begin{array}{c}
\ddot{\phi} \\
\ddot{\theta}_{i}
\end{array}\right]+\left[\begin{array}{c}
F_{1} \\
F_{i}
\end{array}\right]+\left[\begin{array}{c}
G_{1} \\
G_{i}
\end{array}\right]=\left[\begin{array}{c}
\tau_{1} \\
0
\end{array}\right]} \\
\theta_{j}=\theta_{j 0} \quad j \neq 1, i .
\end{gathered}
$$

Here, $\left[H_{k l}(q)\right]$ is the $n \times n$ symmetric positive-definite inertia matrix, $q=\left[\theta_{2}, \ldots, \theta_{n}\right]^{T}$ is the $n-1$ vector of unactuated joint angles, $\left[F_{1}(q, \dot{q}, \dot{\phi}), F_{i}(q, \dot{q}, \dot{\phi})\right]^{T}$ represents the $2 \times 1$ vector of centrifugal and Coriolis effects, and $\left[G_{1}(q, \phi), G_{i}(q, \phi)\right]^{T}$ represents the $2 \times 1$ vector of gravitational effects. The torque on the actuated joint axis $Z_{0}$ is represented by $\tau_{1}$. We note that the $j$ th link $(j \neq 1, i)$ is locked at $\theta_{j 0}$, which is a constant. Using $F_{i}(q, \dot{q}, \dot{\phi})=f_{i}(q) \dot{\phi}^{2}$ and $G_{i}(q, \phi)=g_{i}(q) g \sin \phi$, the second row of (1) may be written as

$$
\ddot{\theta}_{i}=-\frac{H_{1 i}(q)}{H_{i i}(q)} \ddot{\phi}-\frac{f_{i}(q)}{H_{i i}(q)} \dot{\phi}^{2}-\frac{g_{i}(q)}{H_{i i}(q)} g \sin \phi .
$$

As shown in [4], (3) is a second-order nonholonomic constraint and, thus, cannot be integrated to express $\theta_{i}$ as a function of $\phi$. Also, at any given time, only one unactuated link (link $i$ ) is in motion. Thus, the $n$-link problem can be treated as a two-link problem without loss of generality. Hereafter, to simplify the algebra, we deal exclusively with the two-link problem. For the two-link case, we may write (3) as

$$
\ddot{\theta}_{2}=-\frac{H_{12}\left(\theta_{2}\right)}{H_{22}\left(\theta_{2}\right)} \ddot{\phi}-\frac{f_{2}\left(\theta_{2}\right)}{H_{22}\left(\theta_{2}\right)} \dot{\phi}^{2}-\frac{g_{2}\left(\theta_{2}\right)}{H_{22}\left(\theta_{2}\right)} g \sin \phi
$$

where

$$
\begin{aligned}
H_{12}= & M_{2}\left(z_{c 2}+d_{2}\right)\left(y_{c 2} \cos \theta_{2}+\left(x_{c 2}+a_{2}\right) \sin \theta_{2}\right) \\
& +I_{y z 2} \cos \theta_{2}+I_{x z 2} \sin \theta_{2} \\
H_{22}= & I_{z z 2}+M_{2}\left(\left(x_{c 2}+a_{2}\right)^{2}+y_{c 2}^{2}\right) \\
f_{2}= & I_{x y 2} \cos 2 \theta_{2}+0.5\left(I_{y y 2}-I_{x x 2}\right) \sin 2 \theta_{2} \\
& +M_{2}\left(a_{1}+\left(x_{c 2}+a_{2}\right) \cos \theta_{2}-y_{c 2} \sin \theta_{2}\right) \\
& \times\left(\left(x_{c 2}+a_{2}\right) \sin \theta_{2}+y_{c 2} \cos \theta_{2}\right) \\
g_{2}= & -M_{2}\left(\left(x_{c 2}+a_{2}\right) \sin \theta_{2}+y_{c 2} \cos \theta_{2}\right) .
\end{aligned}
$$

Here, $M_{2}$ denotes the mass of link 2. $I_{x y 2}$, etc., denote the moments of inertia of link 2 about a centroidal coordinate frame. The parameters $x_{c 2}, y_{c 2}, z_{c 2}$ are the coordinates of the center of mass of link 2 in the link-attached frame. Also, $a_{2}$ and $d_{2}$ refer to the corresponding Denavit-Hartenberg (D-H) parameters.

As seen in Appendix A, we may choose the control torque $\tau_{1}$ in (1) to converge exponentially to any bounded trajectory for the actuated coordinate $\phi$. We refer to $\phi$ and its derivatives $(\dot{\phi}, \ddot{\phi})$ in (4) as the pseudo-input. The terms involving $\theta_{2}$ in (4), i.e., $H_{12} / H_{22}, f_{2} / H_{22}$, and $g_{2} / H_{22}$, are referred to as the modulating coefficients. These modulating coefficients scale the

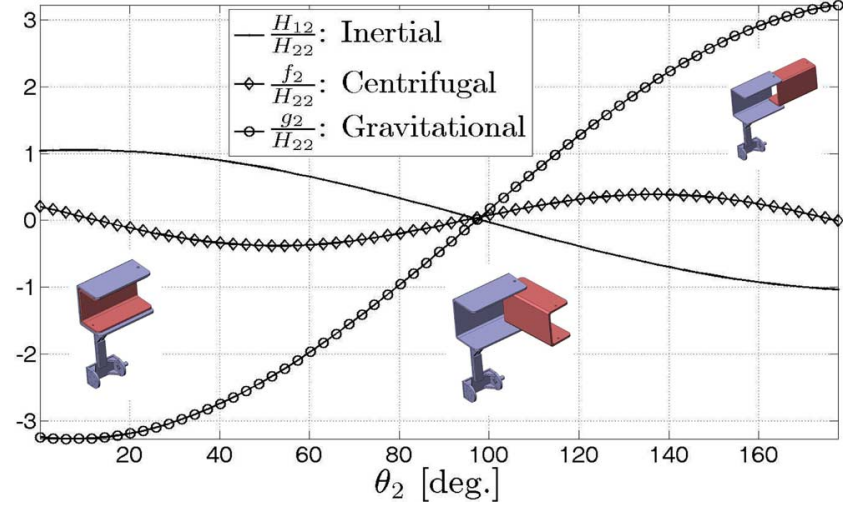

Fig. 4. Comparison of modulating coefficients over configuration space.

various components of the pseudo-input $(\phi, \dot{\phi}, \ddot{\phi})$, depending on the position of the unactuated link 2 .

Fig. 4 shows the variation of the dimensionless modulating coefficients in the configuration space $\left(0^{\circ}-180^{\circ}\right)$ of the unactuated coordinate. The simulation is based on parameter values taken from a two-link version of our prototype system shown later in Fig. 9. The dominant term is the modulating coefficient due to gravity $\left(g_{2} / H_{22}\right)$, followed by the contribution of the inertial coupling $\left(\mathrm{H}_{12} / \mathrm{H}_{22}\right)$ and, finally, the contribution of the centrifugal coupling $\left(f_{2} / H_{22}\right)$. In view of these observations, we make the following assumptions.

1) Inertial coupling is neglected.

2) Centrifugal coupling is neglected.

These assumptions are valid as long as the gravitational component of acceleration $\left|g \sin \theta_{1}\right|$ is of the same (or higher) order of magnitude as compared with $\left|\ddot{\theta}_{1}\right|$ and $\left|\dot{\theta}_{1}^{2}\right|$. We validate these approximations a posteriori in the section on experimental results. Under these assumptions, the dynamics (4) may be simplified as

$$
\ddot{\theta}_{2}=-\frac{g_{2}\left(\theta_{2}\right)}{H_{22}} g \sin \phi .
$$

Using (6) and (8), we may write (9) as

$$
\ddot{\theta}=A \sin \theta \sin \phi
$$

where

$$
\begin{aligned}
\theta & =\theta_{2}+\alpha \\
A & =\frac{M_{2} g \sqrt{y_{c 2}^{2}+\left(x_{c 2}+a_{2}\right)^{2}}}{I_{z z 2}+M_{2}\left(y_{c 2}^{2}+\left(x_{c 2}+a_{2}\right)^{2}\right)} \\
\alpha & =\operatorname{atan} 2\left(y_{c 2}, x_{c 2}+a_{2}\right) .
\end{aligned}
$$

Remark 1: It is worthwhile to examine the physical significance of the dynamics (10). It represents a pendulum in a modulated "gravity" field. For a fixed value of $\phi$, the quantity $A$ may be identified with the square of the natural frequency. The strength of this field can be modulated as $A \sin \phi$ by controlling the angle $\phi$. The pendulum behaves as a regular or inverted pendulum, depending on the $\operatorname{sign}$ of $\sin \theta \sin \phi$. Also, the "gravity" field may be switched off by setting $\phi=0$. This gives rise to a continuum of equilibria given by $[\theta=\bar{\theta}, \dot{\theta}=0, \phi=0]$, where $\bar{\theta}$ is arbitrary. 
Remark 2: Although inertial and centrifugal terms have been neglected in (10), the dynamical system is still second-order nonholonomic. The gravity term is a function of the generalized coordinates $\theta$ and $\phi$. Therefore, it satisfies the conditions for second-order nonholonomic systems stated in [4].

\section{FEEDBACK CONTROL}

\section{A. Control Law}

In this section, we propose a closed-loop control law for point-to-point control of the unactuated link. The goal is to transfer the unactuated link from an initial angular position $\theta_{0}\left(=\theta_{20}+\alpha\right)$ with zero initial velocity to a final angular position $\theta_{f}\left(=\theta_{2 f}+\alpha\right)$ with zero final velocity. We treat the actuated coordinate $\phi$ as a pseudo-input and prescribe a feedback law in terms of the pseudo-input. The formal justification of this treatment is deferred to Appendix A.

From (10), we see that the input $\phi$ has a bounded effect on the acceleration because $|\sin \phi| \leq 1$. We propose a nonlinear feedback control law of the form given by

$$
\sin \phi=\frac{\sin \left(k_{1}\left(\theta_{f}-\theta\right)-\bar{k}_{2} \dot{\theta}\right) \sin \theta}{k}
$$

where $k \geq 1$ and $k_{1}, \bar{k}_{2}>0$ are constants. Also, $\theta_{f}$ is the desired final angular position of the unactuated link. We note that $\phi$ exists because $\left|\sin \left(k_{1}\left(\theta_{f}-\theta\right)-k_{2} \dot{\theta}\right) \sin \theta / k\right| \leq 1$. Using (11) in $(10)$, we get

$$
\ddot{\theta}=\frac{A}{k} \sin \left(k_{1}\left(\theta_{f}-\theta\right)-\bar{k}_{2} \dot{\theta}\right) \sin ^{2} \theta .
$$

Let us introduce a nondimensional time scale $\tau \triangleq \bar{\omega} t$, where $\bar{\omega} \triangleq \sqrt{A / k}$. It may be noted that the parameter $A$ captures the effect of payload and robot arm inertia. The nondimensional time scale makes our subsequent results payload independent. We can now rewrite (12) as

$$
\ddot{\theta}=\sin \left(k_{1}\left(\theta_{f}-\theta\right)-k_{2} \dot{\theta}\right) \sin ^{2} \theta .
$$

Here, $k_{2}=\bar{\omega} \bar{k}_{2}$, and it is understood that the derivatives denote differentiation with respect to $\tau$.

The intuition behind the control law (11) is to introduce a virtual nonlinear spring and damper into the system. These virtual elements introduce a stable equilibrium point $[\theta, \dot{\theta}]=\left[\theta_{f}, 0\right]$ in the system dynamics. In the vicinity of the equilibrium point $\left[\theta_{f}, 0\right]$, the dynamics (13) may be approximated as

$$
\ddot{\theta} \approx\left(k_{1} \sin ^{2} \theta_{f}\right)\left(\theta_{f}-\theta\right)-\left(k_{2} \sin ^{2} \theta_{f}\right) \dot{\theta} .
$$

The parameters $k_{1}$ and $k_{2}$ are measures of stiffness and damping, respectively. Further, the multiplicative term $\sin \theta$ in (11) ensures that the sign of the acceleration $\ddot{\theta}$ in (13) is not affected by the regime of motion ( $\sin \theta>0$ or $\sin \theta<0$ ). It is only affected by the deviation from the desired final state $[\theta, \dot{\theta}]=\left[\theta_{f}, 0\right]$.

The linearized system (14) does not provide any insight about the DOC of the control law for different choices of $k_{1}$ and $k_{2}$. Furthermore, the linearization of (13) about point $(0,0)$, which is the other equilibrium point of the system, leads to a degenerate system. A linearized analysis is inconclusive for such degenerate systems. These issues are formally addressed in the nonlinear convergence analysis below.

\section{B. Proof of Convergence}

Let us consider the domain $\Omega=\left\{[\theta, \dot{\theta}]: \mid k_{1}\left(\theta_{f}-\theta\right)-\right.$ $k_{2} \dot{\theta} \mid \leq \pi / 2$ and $\left.|\theta|<\pi\right\}$ and a Lyapunov function candidate (which is defined on $\Omega$ )

$$
V(\theta, \dot{\theta})=\frac{1}{k_{1}} \int_{0}^{\psi} \sin x \sin ^{2}\left(\frac{x+k_{2} \dot{\theta}}{k_{1}}-\theta_{f}\right) d x+\frac{1}{2} \dot{\theta}^{2}
$$

where $\psi=k_{1}\left(\theta_{f}-\theta\right)-k_{2} \dot{\theta}$.

Proposition: The control law (11) guarantees local asymptotic convergence of the state $[\theta, \dot{\theta}]$ in $(13)$ to $\left[\theta_{f}, 0\right]$ for arbitrary gains $k_{1}, k_{2}>0$. Further, $\exists l\left(k_{1}, k_{2}\right)>0$ for which an estimate of the domain of attraction of the control law is the bounded region $\Omega_{l}=\{[\theta, \dot{\theta}]: V(\theta, \dot{\theta})<l\} \subset \Omega$.

Proof: The scalar function $V(\theta, \dot{\theta})$ defined in (15) is positive definite in $\Omega$ because it satisfies the following conditions.

1) $V\left(\theta_{f}, 0\right)=0$.

2) $V(\theta, \dot{\theta})>0$ in $\Omega \quad \forall[\theta, \dot{\theta}] \neq\left[\theta_{f}, 0\right]$.

The first condition follows from direct substitution in (15) and noting that $[\theta, \dot{\theta}]=\left[\theta_{f}, 0\right]$ implies $\psi=0$. The second condition follows by noting that $\sin x>0$ for $\pi / 2 \geq x>0$ and $\sin x<0$ for $-\pi / 2 \leq x<0$. Thus, for $0<|\psi| \leq \pi / 2$, we have

$$
\int_{0}^{\psi} \sin x \sin ^{2}\left(\frac{x+k_{2} \dot{\theta}}{k_{1}}-\theta_{f}\right) d x>0 .
$$

Henceforth, we abbreviate $V(\theta, \dot{\theta})$ as $V$. It is convenient to rewrite (15) as

$$
V=\left\{\begin{array}{l}
\frac{k_{1}\left(\cos \psi-\cos \left(2 \psi / k_{1}\right)\right) \cos 2 \theta+\left(k_{1} \sin \left(2 \psi / k_{1}\right)-2 \sin \psi\right) \sin 2 \theta}{2\left(k_{1}^{2}-4\right)} \\
+\frac{1}{2 k_{1}}(1-\cos \psi)+\frac{1}{2} \dot{\theta}^{2}, \quad k_{1} \neq 2 . \\
\frac{1}{8}\left(\sin \psi \sin 2 \theta-\psi \sin (\psi+2 \theta)+4 \sin ^{2} \frac{\psi}{2}\right)+\frac{1}{2} \dot{\theta}^{2} \\
\quad k_{1}=2 .
\end{array}\right.
$$

Section III-C shows a construction for $l\left(k_{1}, k_{2}\right)>0$ such that the region $\Omega_{l}=\{[\theta, \dot{\theta}]: V(\theta, \dot{\theta})<l\}$ is a subset of $\Omega$.

The time derivative of (17) along system trajectories is given by

$$
\begin{aligned}
\dot{V}= & \frac{\partial V}{\partial \theta} \dot{\theta}+\frac{\partial V}{\partial \dot{\theta}} \ddot{\theta} \\
= & \left\{\begin{array}{l}
-\frac{k_{2} \sin ^{2} \theta}{k_{1}\left(4-k_{1}^{2}\right)}\left(k_{1} \sin \psi\left(\sin 2 \theta \cos \psi-\sin 2\left(\frac{\psi}{k_{1}}+\theta\right)\right)\right. \\
\left.+\left(2-k_{1}^{2} \sin ^{2} \theta\right) \sin ^{2} \psi\right), \quad k_{1} \neq 2 \\
-\frac{k_{2} \sin ^{2} \theta}{8}\left(\left(1+2 \sin ^{2} \theta\right) \sin ^{2} \psi-\psi \sin \psi \cos (2 \theta+\psi)\right) \\
k_{1}=2
\end{array}\right.
\end{aligned}
$$

Appendix B shows that $\dot{V} \leq 0$ in $\Omega$ for all $k_{1}, k_{2}>0$. Since $\dot{V}$ is only negative semidefinite, we next analyze the invariant sets, where $\dot{V}=0$. From (35) in Appendix B, we have

$$
\dot{V}=0 \Rightarrow \theta=0 \text { or } \psi=0 \text {. }
$$


Using (19) in (12), we get

$$
\dot{V}=0 \Rightarrow \ddot{\theta}=0 \text {. }
$$

From (19) and (20), the largest invariant set, where $\dot{V}=0$, is given by $\left\{[\theta, \dot{\theta}]=[0,0] \cup\left[\theta_{f}, 0\right]\right\}$. Using La Salle's Invariant Set Theorem, we conclude that the state $[\theta, \dot{\theta}]$ converges to $[\theta=0, \dot{\theta}=0]$ or $\left[\theta=\theta_{f}, \dot{\theta}=0\right]$.

It remains to establish the stability of the equilibrium points. If $\theta_{f}=0$, the largest invariant set is $\{[\theta, \dot{\theta}]=[0,0]\}$. Thus, $\left[\theta_{f}, 0\right]$ is a stable equilibrium point. If $\theta_{f} \neq 0$, we show that $[\theta=0, \dot{\theta}=$ $0]$ is unstable and $\left[\theta=\theta_{f}, \dot{\theta}=0\right]$ is a stable equilibrium point. From (17), we have

$$
\left.\frac{\partial^{2} V}{\partial \theta^{2}}\right|_{0,0}=0 \quad \text { and }\left.\quad \frac{\partial^{3} V}{\partial \theta^{3}}\right|_{0,0}=-2 \sin \left(k_{1} \theta_{f}\right) \neq 0 .
$$

This implies that $[\theta=0, \dot{\theta}=0]$ is not a local minimum for $V$ and, thus, an unstable equilibrium point. Once again, from (17), we have

$$
\left.\nabla^{2} V\right|_{\theta_{f}, 0}=\left[\begin{array}{cc}
k_{1} \sin ^{2} \theta_{f} & k_{2} \sin ^{2} \theta_{f} \\
k_{2} \sin ^{2} \theta_{f} & \frac{k_{2}^{2}}{k_{1}} \sin ^{2} \theta_{f}+1
\end{array}\right] .
$$

This implies that $\nabla^{2} V$ is positive definite and $\left[\theta=\theta_{f}, \dot{\theta}=0\right]$ is a local minimum for $V$ and, thus, a stable equilibrium point. Thus, the state $[\theta, \dot{\theta}]$ in $(12)$ converges to $\left[\theta_{f}, 0\right]$ as long as it does not start from $[0,0]$.

\section{Domain of Convergence}

We propose a construction for $l\left(k_{1}, k_{2}\right)>0$ such that the region $\Omega_{l}=\{[\theta, \dot{\theta}]: V(\theta, \dot{\theta})<l\}$ is a subset of $\Omega$. From (17), we have

$$
V=\frac{\sqrt{C^{2}+D^{2}}}{2\left(k_{1}^{2}-4\right)} \sin (2 \theta+E)+\frac{1}{2 k_{1}}(1-\cos \psi)+\frac{1}{2} \dot{\theta}^{2}
$$

where $C=k_{1}\left(\cos \psi-\cos \left(2 \psi / k_{1}\right)\right), \quad D=k_{1} \sin \left(2 \psi / k_{1}\right)-$ $2 \sin \psi$, and $E=a \tan 2(C, D)$. From (15) and (21), we have

$$
\begin{aligned}
& \frac{1}{k_{1}} \int_{0}^{\psi} \sin x \sin ^{2}\left(\frac{x+k_{2} \dot{\theta}}{k_{1}}-\theta_{f}\right) d x \\
& \quad=\frac{\sqrt{C^{2}+D^{2}}}{2\left(k_{1}^{2}-4\right)} \sin (2 \theta+E)+\frac{1-\cos \psi}{2 k_{1}} .
\end{aligned}
$$

Using (16) in (22) and noting that $|\sin (2 \theta+E)| \leq 1$, we have

$$
\frac{1-\cos \psi}{2 k_{1}}-\frac{\sqrt{C^{2}+D^{2}}}{2\left|k_{1}^{2}-4\right|} \geq 0 .
$$

From (21) and (23), we have

$$
V \geq \bar{V} \triangleq \frac{1}{2 k_{1}}(1-\cos \psi)(1-|\sin (2 \theta+E)|)+\frac{1}{2} \dot{\theta}^{2} .
$$

Let us consider the region (see Fig. 5)

$$
\bar{\Omega}_{l}=\{[\theta, \dot{\theta}]: \bar{V}(\theta, \dot{\theta})<l\}
$$

where

$$
l=\frac{1}{2 k_{1}}\left(1-\cos \psi_{\max }\right)\left(1-\left|\sin \gamma_{\max }\right|\right) .
$$

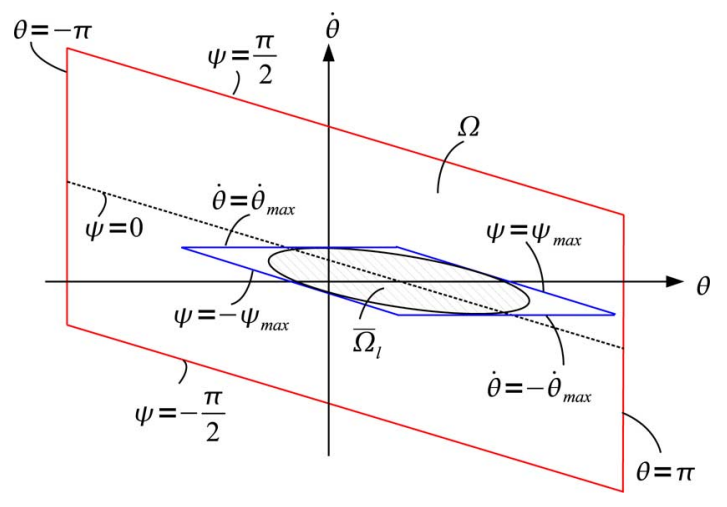

Fig. 5. Choice of $l$ to establish DOC.

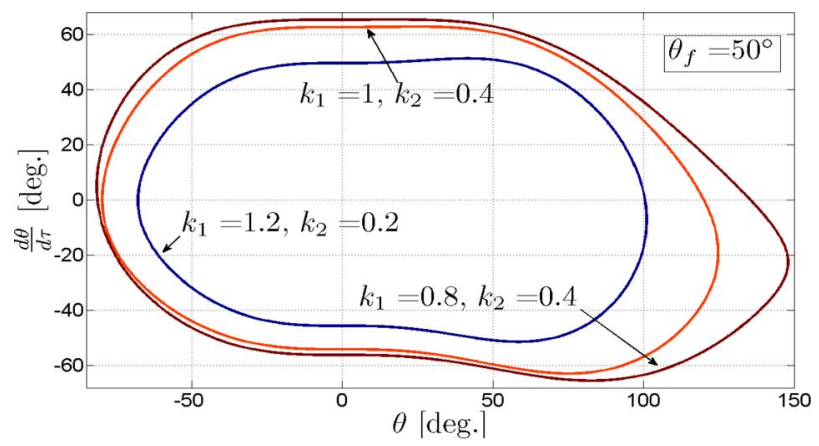

(a)

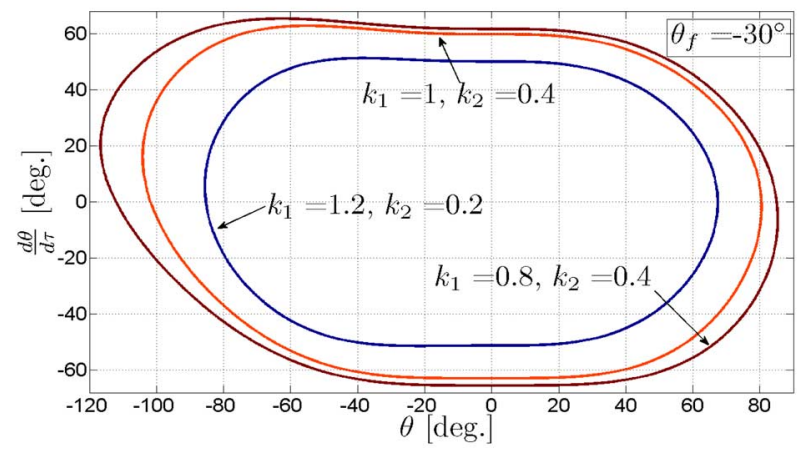

(b)

Fig. 6. Estimates of DOC for various gains and final positions. (a) Final position $50^{\circ}$. (b) Final position $-30^{\circ}$.

Here, $\psi_{\max } \in(0, \pi / 2)$ and $\gamma_{\max } \in(0, \pi / 2)$ or $(\pi, 3 \pi / 2)$ are to be determined such that $|\theta| \leq \pi$ and $|2 \theta+E| \in\left[0, \gamma_{\max }\right]$ or $\left[\pi, \gamma_{\max }\right]$. From (24) to (26), we have

$$
\psi, \dot{\theta} \in \bar{\Omega}_{l} \Rightarrow|\psi| \leq \psi_{\max } \quad \text { and } \quad|\dot{\theta}|<\dot{\theta}_{\max } \triangleq \sqrt{2 l} .
$$

Condition (27) implies that $\bar{\Omega}_{l} \subset \Omega$. From (24), $V \geq \bar{V}$. Thus, $\Omega_{l} \subset \bar{\Omega}_{l} \subset \Omega$.

Remark 3: Fig. 6(a) shows a comparison of the estimates of the DOC for various choices of $k_{1}$ and $k_{2}$ and desired final position of $\theta_{f}=50^{\circ}$. Fig. 6(b) shows a similar comparison for $\theta_{f}=-30^{\circ}$. It may be noted that the DOC is a function of the control gains as well as the final position.

Remark 4: The DOC estimates in Fig. 6 represent sufficient conditions for convergence. Fig. 7 shows three system 


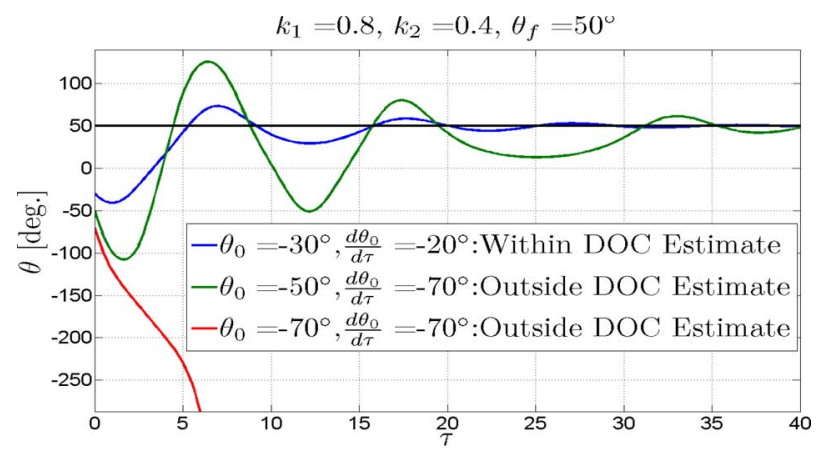

Fig. 7. System trajectories for various initial conditions showing sufficient nature of DOC estimate.

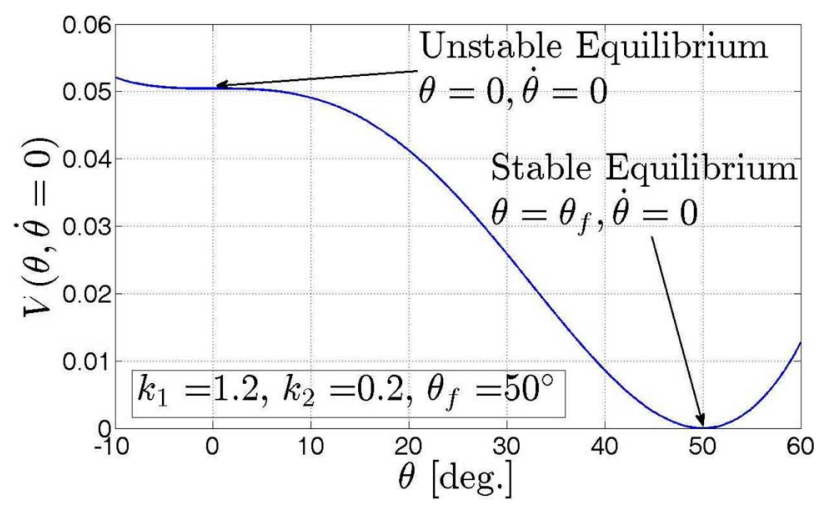

Fig. 8. Stable and unstable equilibria of system dynamics.

trajectories, one of which $\left(\theta_{0}=-50^{\circ}, d \theta_{0} / d \tau=-70^{\circ}\right)$ converges despite starting outside the DOC estimate. The other two trajectories are in accordance with the DOC estimate shown in Fig. 6(a).

Remark 5: It may be noted that the instability of the equilibrium point $[\theta=0, \dot{\theta}=0]$ does not follow from linearization because the linearized system has zero eigenvalues at that point. Fig. 8 shows the variation of the Lyapunov function as a function of $\theta$ on the plane $\dot{\theta}=0$. The parameter values were $k_{1}=1.2$, $k_{2}=0.2$, and $\theta_{f}=50^{\circ}$. The equilibrium $[\theta=0, \dot{\theta}=0]$ is unstable, while the destination $\left[\theta=\theta_{f}, \dot{\theta}=0\right]$ is stable.

\section{IMPLEMENTATION AND EXPERIMENTS}

We conducted position control experiments on a prototype system with three links that is shown in Fig. 9. The link mechanism, which operates inside the wing box, is shown in Fig. 9(a). The links are essentially C-channels that are serially connected by 1-DOF rotary joints. Link 1 is the only servoed link. Links 2 and 3 are equipped with ON-OFF pneumatic brakes with a braking torque of $20 \mathrm{~N} \cdot \mathrm{m}$ each. The relative angular displacement of each link is measured using an optical encoder placed at the rotary joint. The resolution of each encoder is 1000 pulses per revolution.

The actuators and transmission mechanisms for link 1 are completely outside the wing box and are shown in Fig. 9(b). They comprise a servoed tilting mechanism and a servoed azimuthal positioning mechanism. The tilting mechanism is used

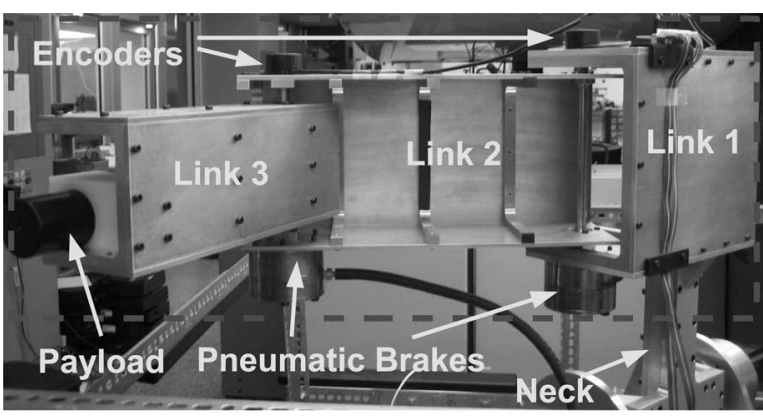

(a)

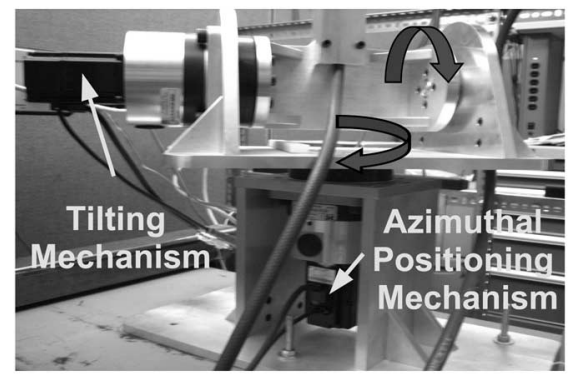

(b)

Fig. 9. Three-link prototype arm. (a) Link mechanism (operates inside wing-box). (b) Actuation mechanisms (operate outside wing-box).

TABLE 1

INERTIA AND D-H PARAMETERS OF ROBOT ARM

\begin{tabular}{|c||c|c|c|}
\hline & Link 1 & Link 2 & Link 3 \\
\hline $\mathrm{M}(\mathrm{kg})$ & 8.47 & 6.80 & 1.89 \\
\hline$I_{x x}\left(\mathrm{~kg}-\mathrm{m}^{2}\right)$ & 0.27 & 0.06 & 0.01 \\
\hline$I_{x y}\left(\mathrm{~kg}-\mathrm{m}^{2}\right)$ & 0.00 & 0.01 & 0.00 \\
\hline$I_{x z}\left(\mathrm{~kg}-\mathrm{m}^{2}\right)$ & 0.03 & -0.03 & 0.00 \\
\hline$I_{y y}\left(\mathrm{~kg}-\mathrm{m}^{2}\right)$ & 0.22 & 0.16 & 0.02 \\
\hline$I_{y z}\left(\mathrm{~kg}-\mathrm{m}^{2}\right)$ & -0.03 & -0.01 & 0.00 \\
\hline$I_{z z}\left(\mathrm{~kg}-\mathrm{m}^{2}\right)$ & 0.09 & 0.12 & 0.02 \\
\hline $\mathrm{a}(\mathrm{m})$ & 0.04 & 0.34 & 0.00 \\
\hline $\mathrm{d}(\mathrm{m})$ & 0.18 & 0.29 & 0.32 \\
\hline
\end{tabular}

to tilt link 1 relative to a vertical axis. Depending on the state (ON or OFF) of the pneumatic brakes, the unactuated links ( 2 and 3 ) may be deployed by exploiting gravity and dynamic coupling with link 1. The azimuthal positioning mechanism is used for angular positioning of the entire link mechanism inside the wing box and serves to expand the workspace of the robot arm. This mechanism is used after the links have been deployed using the tilting mechanism. The pneumatic brakes are in the ON state when the azimuthal positioning mechanism is in use. Both mechanisms have harmonic drive gearing $(100: 1)$ coupled with ac servomotors $(0.64 \mathrm{~N} \cdot \mathrm{m}, 3000 \mathrm{r} / \mathrm{min})$. In the experiments that follow, the azimuthal positioning mechanism is not used. We only use the tilting mechanism to deploy the links and verify the proposed control law.

The dynamical system (10) corresponding to our experimental setup has the parameters $A=32.8 \mathrm{~s}^{-2}$ and $\alpha=-3.22^{\circ}$. The inertia and D-H parameters of the robot arm are shown in Table I. The experimental results are shown in Fig. 10. The goal was to move link 2 from an initial position $\theta_{20}=35^{\circ}$ to a desired final position of $\theta_{2 f}=50^{\circ}$. Link 3 was kept fixed at $30^{\circ}$ relative to 


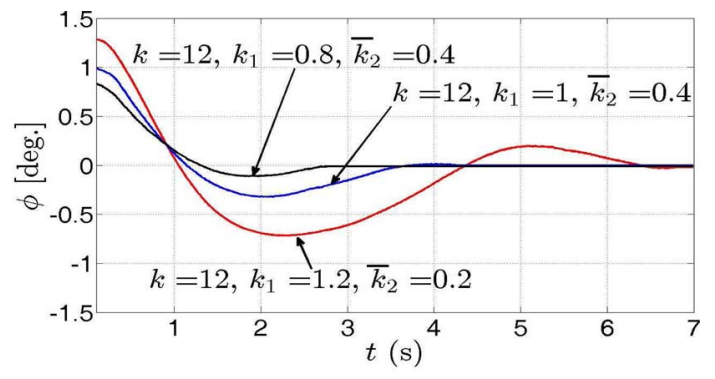

(a)

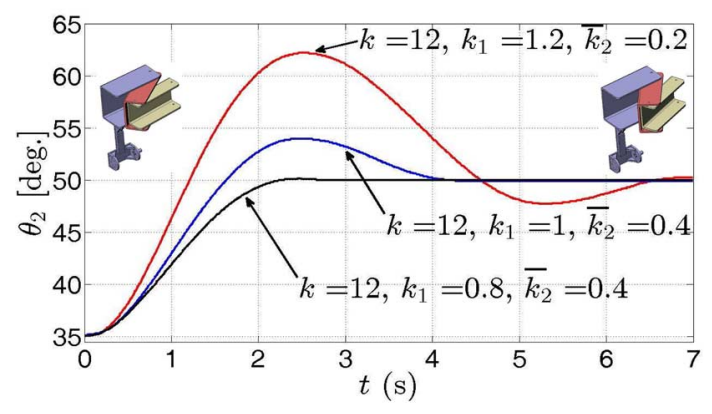

(b)

Fig. 10. Position control experiment on three-link prototype. (a) Link 1 trajectory (servoed joint). (b) Link 2 trajectory (unactuated joint).

link 2. The experiments were conducted for the following three sets of gains.

1) $k=12, k_{1}=1.2$, and $\bar{k}_{2}=0.2 \mathrm{~s}$.

2) $k=12, k_{1}=1$, and $\bar{k}_{2}=0.8 \mathrm{~s}$.

3) $k=12, k_{1}=0.8$, and $\bar{k}_{2}=0.4 \mathrm{~s}$.

It may be verified that these controller parameters ensure that the initial position lies within the estimated DOC for the desired final position $\theta_{2 f}=50^{\circ}$.

The tilt trajectory $(\phi)$ of link 1 is shown in Fig. 10(a) for various choices of the control gains. A small amplitude of $\phi$ is very important in practice because the arm operates inside an aircraft wing. If link 2 starts at $\theta_{0}$ with zero initial velocity, the initial tilt of link 1 is given by

$$
\sin \phi_{0}=\frac{\sin \left(k_{1}\left(\theta_{f}-\theta_{0}\right)\right) \sin \theta_{0}}{k}
$$

The maximum tilt is $1.3^{\circ}$ for the case $k=12$ and $k_{1}=1.2$. This is small enough for operation inside the wing box.

The trajectory $\left(\theta_{2}\right)$ of the free link is shown in Fig. 10(b). As before, we gather insight into the nonlinear system (13) through the behavior of the linearized system (14). From (14), the quantities $\omega_{\text {lin }} \equiv \sqrt{k_{1}} \sin \theta_{f}$ and $\zeta_{\text {lin }} \equiv k_{2} \sin \theta_{f} / 2 \sqrt{k_{1}}$ may be interpreted as measures of "natural frequency" and "damping ratio," respectively. As expected, less overshoot is observed as the "damping ratio" is increased. A lower "damping ratio" also results in a faster rise time. However, the corresponding settling time is also larger because of oscillations. As seen in Fig. 10(b), the convergence time was $t_{f}=6.5 \mathrm{~s}, t_{f}=4 \mathrm{~s}$, and $t_{f}=2.5 \mathrm{~s}$ for the various control gains. It may be noted that the pneumatic brake was released at $t=0$ and reactivated at $t=t_{f}$.

Fig. 11 shows a comparison of the gravitational, inertial, and centrifugal contributions to the angular acceleration of link 2 for
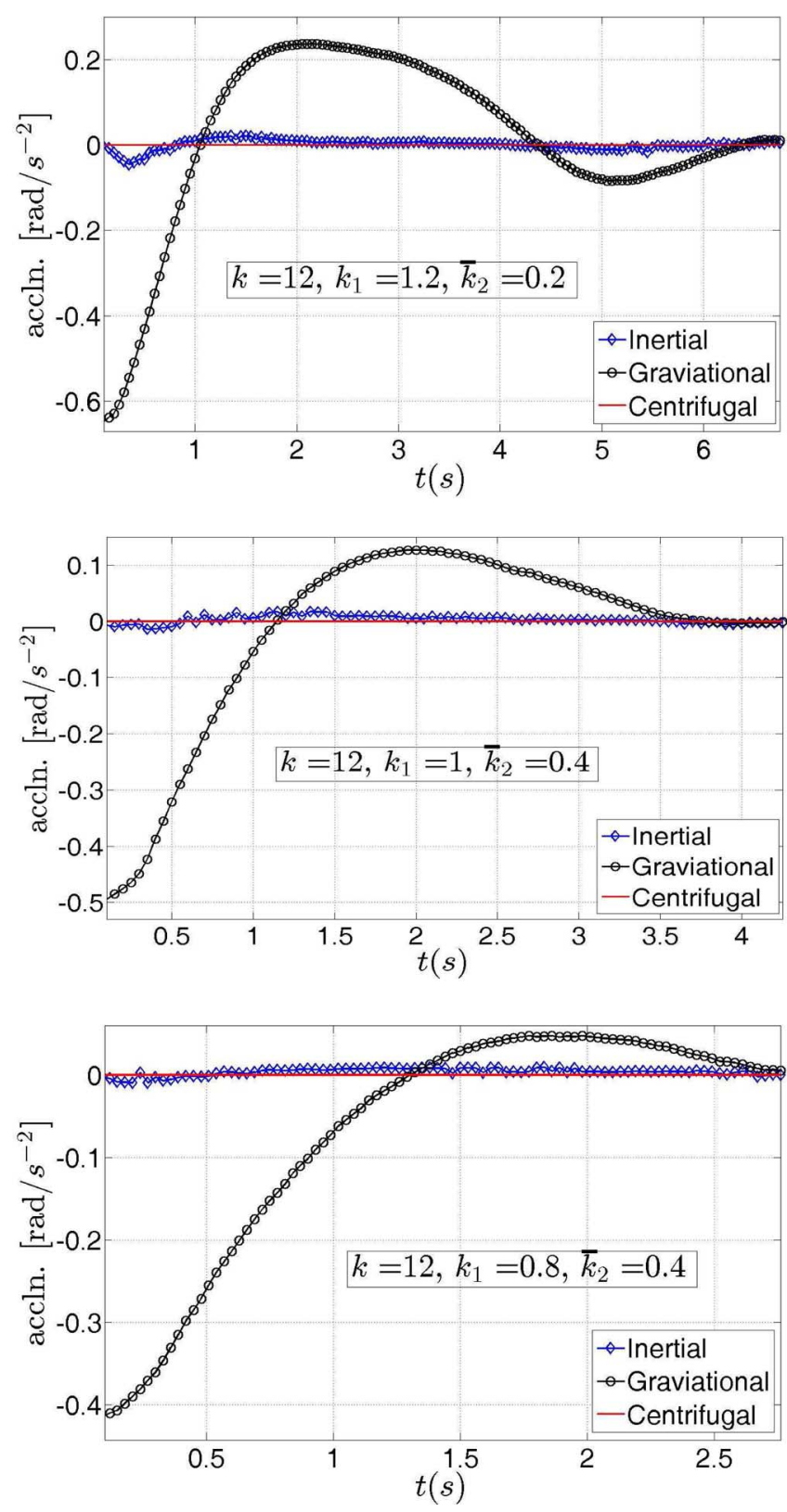

Fig. 11. Comparison of gravitational, inertial, and centrifugal contributions to acceleration of free link.

each set of control gains. The gravitational contribution clearly dominates the other dynamical effects in each case. This demonstrates, a posteriori, the validity of the approximations made in our dynamic modeling.

The control law (11) demonstrates reasonable positioning accuracy of the unactuated links. The performance is achieved without any knowledge of stiction or the dynamics introduced by the flexible hose supplying air to the pneumatic brakes. This is a significant improvement compared with the open-loop motion-planning schemes explored in our earlier work [21]. Such schemes required frequent tuning of friction coefficients and other parameters related to the dynamics of the hose.

Remark 6: In practice, the gains $\left(k, k_{1}, k_{2}\right)$ must be chosen for fastest convergence $\left(1 / \zeta_{\operatorname{lin}} \omega_{\text {lin }}\right)$ such that the initial position 
is within the DOC (from Lyapunov function) and the maximum tilt of the actuated joint is within limits.

\section{CONCLUSION}

We have addressed the problem of closed-loop point-topoint control of a gravity-assisted underactuated robot arm with second-order nonholonomic constraints. The joints of the hyperarticulated arm are actuated by gravity and locked with pneumatic ON-OFF brakes. By tilting the base link appropriately, the gravitational torque drives the unactuated links to a desired angular position. The hyperarticulated arm can change its configuration using only one actuator at the base in conjunction with the ON-OFF brakes. This underactuated arm design was motivated by the need for a compact snake-like robot that can go into aircraft wings and perform assembly operations using heavy end-effectors.

We explored the system dynamics to distil a simple model that captures the dominant dynamical effects. We proposed a nonlinear closed-loop control algorithm for point-to-point control of the unactuated links. A Lyapunov function was synthesized to prove the convergence of the control law. The Lyapunov function was also used to generate estimates of the DOC of the control law for various control gains. The control algorithm was applied to a prototype three-link robot arm. The experimental results showed reasonable performance of the control law in the absence of prior knowledge of friction and other unmodeled dynamical effects.

This study focused on sequential deployment of the unactuated links for desired end-point positioning. The authors are currently investigating control techniques for concurrent deployment of multiple links.

\section{APPENDIX A}

We justify the treatment of the actuated coordinate $\phi$ as a pseudoinput. We denote the desired trajectory of $\phi$ by $\phi_{d}$. From (11), $\phi_{d}=\sin ^{-1}\left(\sin \left(k_{1}\left(\theta_{f}-\theta\right)-k_{2} \dot{\theta}\right) \sin (\theta / k)\right)$. The dynamics of the actuated coordinate $\phi$ may always be feedback linearized by choosing the control torque as

$$
\tau_{1}=\frac{\ddot{\phi}_{d}-2 \lambda \dot{\tilde{\phi}}-\lambda^{2} \tilde{\phi}}{N_{11}}+F_{1}+G_{1}+\frac{N_{12}}{N_{11}}\left(F_{i}+G_{i}\right)
$$

where

$$
\begin{aligned}
{\left[\begin{array}{cc}
N_{11} & N_{i 1} \\
N_{i 1} & N_{i i}
\end{array}\right] } & =\left[\begin{array}{ll}
H_{11} & H_{i 1} \\
H_{i 1} & H_{i i}
\end{array}\right]^{-1} \\
\tilde{\phi} & =\phi-\phi_{d}, \quad \text { and } \quad \lambda>0 .
\end{aligned}
$$

Using (29) in (1), the error dynamics of the actuated coordinate is given by

$$
\ddot{\tilde{\phi}}+2 \lambda \dot{\tilde{\phi}}+\lambda^{2} \tilde{\phi}=0 .
$$

Let us define $x=[\theta, \dot{\theta}]^{T}$ and $y=[\tilde{\phi}, \dot{\tilde{\phi}}]^{T}$. The dynamics of the unactuated coordinate $(x)$ and the error dynamics of the actuated coordinate $(y)$ may be written in cascade form as $\dot{x}=f(x, y)$ and $\dot{y}=g(y)$. Here, $f(x, y)=\left[\dot{\theta}, A \sin \theta \sin \left(\phi_{d}+\tilde{\phi}\right)\right]^{T}$, and $g(y)=\left[\dot{\tilde{\phi}},-2 \lambda \dot{\tilde{\phi}}-\lambda^{2} \tilde{\phi}\right]^{T}$. We note that $f(x, y)$ is globally Lipschitz and the linear subsystem $\dot{y}=g(y)$ is globally exponentially stable. Also, we have proved that the nonlinear subsystem $\dot{x}=f(x, 0)$ is asymptotically stable using La Salle's Theorem. It follows from Sontag's Theorem [23], [24] that the cascade system is locally asymptotically stable for an appropriate choice of $\lambda$.

\section{APPENDIX B}

We establish the negative semidefiniteness of $\dot{V}$ for all $k_{1}, k_{2}>0$. Let us define

$$
\begin{aligned}
F_{k_{1}}(\theta, \psi) & \triangleq(P+R) \tan ^{2} \theta+2 Q \tan \theta+R \\
P & \triangleq k_{1}^{2} \sin ^{2} \psi-2 k_{1} \sin \psi \sin \frac{2 \psi}{k_{1}} \\
Q & \triangleq k_{1} \sin \psi\left(\cos \frac{2 \psi}{k_{1}}-\cos \psi\right) \\
R & \triangleq k_{1} \sin \psi \sin \frac{2 \psi}{k_{1}}-2 \sin ^{2} \psi .
\end{aligned}
$$

Using (31), we may rewrite (18) as

$$
\dot{V}=-\frac{k_{2} \sin ^{2} \theta \cos ^{2} \theta}{k_{1}\left(k_{1}^{2}-4\right)} F_{k_{1}}(\theta, \psi) .
$$

We consider three cases to prove the negative semidefiniteness of $\dot{V}$ in $\Omega$.

1) $k_{1}>2$ : In this case, we prove that $F_{k_{1}}(\theta, \psi) \geq 0$. We note that, for $k_{1}>2$, we have $P, R \geq 0$. Let us define

$$
S_{k_{1}}(\psi) \triangleq(P+R) R-Q^{2} .
$$

Thus, $F_{k_{1}}(\theta, \psi) \geq 0$ iff $S_{k_{1}}(\psi) \geq 0$. Substituting (32)(34) into (36), we have

$$
\begin{aligned}
S_{k_{1}}(\psi)= & k_{1}^{3} \sin \psi \sin \frac{2 \psi}{k_{1}}+2 k_{1}^{2} \cos \frac{2 \psi}{k_{1}} \cos \psi \\
& -\left(k_{1}^{2}-4\right) \sin ^{2} \psi-2 k_{1}^{2} .
\end{aligned}
$$

We note that $S_{k_{1}}(0)=0$ and $S_{k_{1}}(\psi)=S_{k_{1}}(-\psi)$. In addition

$$
\begin{gathered}
\frac{\partial S_{k_{1}}}{\partial \psi}=\left(k_{1}^{2}-4\right)\left(k_{1} \sin \frac{2 \psi}{k_{1}}-2 \sin \psi\right) \cos \psi \geq 0, \\
0 \leq \psi \leq \pi / 2 .
\end{gathered}
$$

Thus, $S_{k_{1}}(\psi) \geq 0$ for $|\psi| \leq \pi / 2$.

2) $0<k_{1}<2$ : In this case, we prove that $F_{k_{1}}(\theta, \psi) \leq 0$. The argument follows along the same lines noting that $R \leq 0, P+R \leq 0$, and $S_{k_{1}}(\psi) \geq 0$.

3) $k_{1}=2$ : In this case, we prove that

$$
\begin{aligned}
f_{2}(\theta, \psi) & \triangleq \lim _{k_{1} \rightarrow 2} \frac{F_{k_{1}}(\theta, \psi)}{k_{1}-2} \\
& =\left(p_{2}+r_{2}\right) \tan ^{2} \theta+2 q_{2} \tan \theta+r_{2} \geq 0
\end{aligned}
$$

where $p_{2} \triangleq \lim _{k_{1} \rightarrow 2} P /\left(k_{1}-2\right), q_{2} \triangleq \lim _{k_{1} \rightarrow 2} Q /$ $\left(k_{1}-2\right)$, and $r_{2} \triangleq \lim _{k_{1} \rightarrow 2} R /\left(k_{1}-2\right)$. The argument 
follows along the same lines noting that $p_{2} \geq 0, r_{2} \geq 0$, and $s_{2}(\psi) \triangleq \lim _{k_{1} \rightarrow 2} S_{k_{1}}(\psi) /\left(k_{1}-2\right) \geq 0$.

\section{ACKNOWLEDGMENT}

The first author would like to thank Dr. J. Ueda for many helpful discussions.

\section{REFERENCES}

[1] B. Roy and H. Asada, "An underactuated robot with a hyper-articulated deployable arm working inside an aircraft wing-box," in Proc. IEEE/RSJ Int. Conf. Intell. Robots Syst. (IROS), Aug. 2-6, 2005, pp. 4046-4050.

[2] I. Kolmanovsky and N. H. McClamroch, "Developments in nonholonomic control problems," IEEE Control Syst. Mag., vol. 15, no. 6, pp. 20-36, Dec. 1995.

[3] R. M. Murray, L. Zexiang, and S. S. Sastry, A Mathematical Introduction to Robotic Manipulation, 1st ed. Boca Raton, FL: CRC, 1994.

[4] G. Oriolo and Y. Nakamura, "Free-joint manipulators: Motion control under second-order nonholonomic constraints," in Proc. IEEE/RSJ Int. Workshop Intell. Robots Syst., Nov. 3-5, 1991, pp. 1248-1253.

[5] H. Arai, K. Tanie, and N. Shiroma, "Nonholonomic control of a three-dof planar underactuated manipulator," IEEE Trans. Robot. Autom., vol. 14, no. 5, pp. 681-695, Oct. 1998.

[6] K. M. Lynch, N. Shiroma, H. Arai, and K. Tanie, "Collision-free trajectory planning for a 3-dof robot with a passive joint," Int. J. Robot. Res., vol. 19, no. 12 , pp. 1171-1184, 2000.

[7] A. D. Luca, R. Mattone, and G. Oriolo, "Stabilization of an underactuated planar 2r manipulator," Int. J. Robust Nonlinear Control, vol. 10, pp. 181198, 2000.

[8] K. Kobayashi and T. Yoshikawa, "Trajectory planning and control for planar robots with passive last joint," Int. J. Robot. Res., vol. 21, pp. 575$590,2002$.

[9] A. D. Luca and G. Oriolo, "Motion planning under gravity for underactuated three-link robots," in Proc. IEEE/RSJ Int. Conf. Intell. Robots Syst. (IROS), Oct. 31-Nov. 05, 2000, pp. 139-144.

[10] K. Kobayashi and T. Yoshikawa, "Controllability of under actuated planar manipulators with one unactuated joint," Int. J. Robot. Res., vol. 21, pp. 555-561, 2002.

[11] T. Suzuki, M. Koinuma, and Y. Nakamura, "Chaos and nonlinear control of a nonholonomic free-joint manipulator," in Proc. IEEE Int. Conf. Robot. Autom., Apr. 22-28, 1996, pp. 2668-2675.

[12] T. Suzuki and Y. Nakamura, "Control of manipulators with free-joints via the averaging method," in Proc. IEEE Int. Conf. Robot. Autom., Apr. 20-25, 1997, pp. 2998-3005.

[13] T. Suzuki, W. Miyoshi, and Y. Nakamura, "Control of 2r underactuated manipulator with friction," in Proc. 37th IEEE Conf. Decision Control, Dec. 16-18, 1998, pp. 2007-2012.

[14] Y. Nakamura, T. Suzuki, and M. Koinuma, "Nonlinear behavior and control of a nonholonomic free-joint manipulator," IEEE Trans. Robot. Autom., vol. 13, no. 6, pp. 853-862, Dec. 1997.

[15] T. Suzuki, "Anti-gravity control of free-joint manipulators by vibrational input," in Proc. IEEE Int. Conf. Robot. Autom., vol. 2, 2002, pp. 13151320.

[16] Y. Nakamura and R. Mukherjee, "Nonholonomic path planning of space robots via a bidirectional approach," IEEE Trans. Robot. Autom., vol. 7, no. 4, pp. 500-514, Aug. 1991

[17] R. Mukherjee and D. Chen, "Control of free-flying underactuated space manipulators to equilibrium manifolds," IEEE Trans. Robot. Autom., vol. 9 , no. 5, pp. 561-570, Oct. 1993.

[18] E. Papadopoulos and S. Dubowsky, "The kinematics, dynamics, and control of free-flying and free-floating space robotic systems," IEEE Trans. Robot. Autom., vol. 9, no. 5, pp. 531-543, Oct. 1993.

[19] J. Hauser and R. M. Murray, "Nonlinear controller for nonintegrable systems: The acrobot example," in Proc. Amer. Control Conf., Nov. 3-5, 1990, pp. 669-671.
[20] M. W. Spong, "Swing up control of the acrobot," IEEE Control Syst. Mag., vol. 15, no. 5, pp. 49-55, Feb. 1995.

[21] B. Roy and H. Asada, "Dynamics and control of a gravity-assisted underactuated robot arm for assembly operations inside an aircraft wing-box," in Proc. IEEE Int. Conf. Robot. Autom., Orlando, FL, May 2006, pp. $2007-$ 2012.

[22] B. Roy and H. Asada, "Closed loop control of a gravity-assisted underactuated robot arm with application to aircraft wing-box assembly," presented at the Robot.: Sci. Syst. Atlanta, GA, Jun. 2007.

[23] E. D. Sontag, "Remarks on stabilization and input-to-state stability," in Proc. 28th IEEE Conf. Decision Control, Dec. 13-15, 1989, pp. 13761378.

[24] H. J. Sussmann and P. Kokotovic, "The peaking phenomenon and the global stabilization of nonlinear systems," IEEE Trans. Automat. Control, vol. 36, no. 4, pp. 424-440, Apr. 1991.

[25] H. J. Sussmann, "A general theorem on local controllability," SIAM J. Control Optim., vol. 25, no. 1, pp. 158-194, 1987.

[26] J. M. Hollerbach, "Dynamic scaling of manipulator trajectories," Trans. ASME, J. Dyn. Syst. Meas. Control, vol. 106, pp. 102-106, 1984.

[27] E. Papadopoulos and S. Dubowsky, "On the nature of control algorithms for free-floating space manipulators," IEEE Trans. Robot. Autom., vol. 7, no. 6, pp. 750-758, Dec. 1991.

[28] R. W. Brockett, "Asymptotic stability and feedback stabilization," in Differential Geometric Control Theory, R. S. M. R. W. Brockett and H. J. Sussmann, Eds. Boston, MA: Birkhauser, 1983, pp. 181-191.

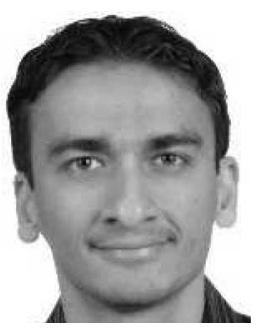

Binayak Roy received the B.Tech. degree in manufacturing science and engineering in 2001 from the Indian Institute of Technology Kharagpur, Kharagpur, India, and the S.M and Ph.D. degrees in mechanical engineering from the Massachusetts Institute of Technology (MIT), Cambridge, in 2003 and 2008, respectively.

He is currently with MIT. His current research interests include engineering design, robotics, and the control of nonliner dynamical systems.

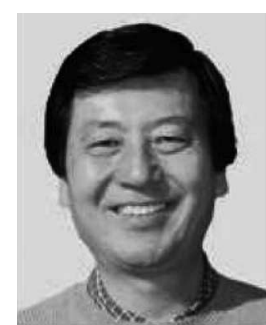

H. Harry Asada (M'06) received the B.S. degree in mechanical engineering in 1973 and the M.S. and $\mathrm{Ph} . \mathrm{D}$. degrees in precision engineering in 1975 and 1978, respectively, all from Kyoto University, Kyoto, Japan.

He was a Research Scientist with the Robotics Institute, Carnegie-Mellon University, Pittsburgh, PA. In 1982, he joined the Massachusetts Institute of Technology (MIT), Cambridge, as a Faculty Member. From 1985 to 1988 , he was a Faculty Member with the Department of Applied Mathematics and Physics, Kyoto University. Since 1990, he has been a Full Professor with the Department of Mechanical Engineering, MIT. His current research interests include robotics and control, biomedical engineering, system dynamics, engineering design, manufacturing, and communication systems.

Dr. Asada received the O. Hugo Shuck Best Paper Award in 1984 from the American Control Council, three Best Paper Awards at the IEEE Robotics and Automation Conference in 1993, 1997, and 1999, and five Best Journal Papers Awards from the Journal of Advanced Robotics, the Journal of Instrument and Control Engineering, and the Journal of Systems and Control. He received the 1998 American Society of Mechanical Engineers (ASME) Dynamic Systems and Control Outstanding Researcher Award and is a Fellow of ASME. 